\title{
Design in line LMA-10 Photonic Crystal Fiber MZI for Detection and investigation of Beta Thalassemia
}

\author{
Layla M. Alameri ${ }^{1}$, Tahreer S. Mansour ${ }^{1}$, Fareed F. Rashid ${ }^{2}$, Sara J. Belal ${ }^{2}$ \\ ${ }^{1}$ Institute of Laser for Postgraduate Studies, University of Baghdad., Baghdad, Iraq, \\ ${ }^{2}$ Fareed F. Rashid and Sara J. Belal are with the Department of Laser and Optoelectronics Engineering from \\ University of Technology, Baghdad, Iraq
}

\begin{abstract}
Consequent to the scarcity of removable rapid diagnostic examines for detecting the thalassemia, specially beta thalassemia trait or Cooly's Anemia, present detection methods include the collection of the blood, which then required being more tests before reports can be made by the treating physicians.Traditional methods like hemoglobinopathy tests and blood picture are time expending, needing specialized technical personnel, extra huge and costly laboratory instrument. Here, we simulated and demonstrated micro- hole collapsing, type of Mazh-Zehnder interferometer (MZI) that formed using LMA-! 0 fiber, with a laser beam for detection the most common type of anemia, beta thalassemia trait or Cooly's Anemia .this interferometer made-up of two conventional optical fibers splicing to apart of photonic crystal fiber (PCF).this method makes a high sensitive regions for the optical characteristics in the blood sample. Biological changes in the blood sample consequent to the presence the beta thalassemia trait lead to changes optical characteristics (refractive index and absorption) of the blood sample the demonstrated laser biosensor has the ability for use as a removable, rapid diagnosis examines and the high sensitive which rises to $24.367 \mathrm{ABS} / \mathrm{RIU}$.
\end{abstract}

Keywords: Beta Thalassemia; Crystal Fiber MZI; LMA-10 Photonic

\section{Introduction}

DUE TO the special characteristics of optical fibers, they are used in a wide variety of applications specially as a biosensor for high precision measurement ${ }^{(1)}$. These have a large role in different biological applications after production a new design of these fibers, fiber Bragg grating and photonic crystal fiber, ${ }^{(2,3)}$. Designing Laser biosensor needs a new modification of fiber core either using a new material to produce a fiber Bragg grating (FBG) or by using a new fiber cladding that has many holes arranged in a honey comb with either hollow or silica core ${ }^{(4-7)}$. Different techniques must be used for producing in line fiber sensor that are scraping, cleaving and fusion splicing either solid or hollow cores photonic crystal fiber may be with 19 or 7 cells ${ }^{(8,9,10)}$. Laser biosensors have a many roles in different fields, such as immunoassays and drug detection due to their high precision and sensitivity ${ }^{(11,12)}$. In general, optical sensors can be distributed into four classes based on changing in light parameters such as polarization modulation intensity modulation, wavelength modulation, and phase modulation.
Intensity modulation was used in early optical sensor development due to its low cost, simplicity, and reliability $(13,14)$. This intensity modulated signal comes from Fiber Bragg grating as a sensing head that may be used as optical band pass or notch filters ${ }^{(15,17)}$.The traditional tests of beta thalassemia trait is by hemoglobinopathy analysis and complete genotype which identified the beta thalassemia trait .Beta thalassemia trait also is an important manner for decreasing the expression of $\mathrm{HbA} 2$ with level as low as $3.6 \%$ on high performance liquid chromatography (HPLC).This type of anemia needs regular blood transfusions and special medical care through their life. The beta thalassemia trait is the most widespread anemia in the south chines populations. This type of anemia may be not easy to do correct diagnostic who examined on HPLC ${ }^{(18,19)}$. In this study, we present invasive beta thalassemia trait detection biosensor using in-line PCF MZI and laser beam.This type of sensor depends on light intensity measurement through a modified optical fiber. 
LASER BIOSENSOR BASED ON MICOHOLE COLLAPSING METHODS: DESIGN AND PRINCIPLE

At the begging, single mode fiber (SMF-28) had been spliced with an inexpensive $1.5 \mathrm{~cm}$ solid core photonic crystal fiber length, LMA-10, with cladding diameter: $125 \mu \mathrm{m}$ and core diameter: $7.0 \mu \mathrm{m}$. The cross section of the PCF (LMA-10).

The splicing was done by using Fujikura (FSM-60S) splicing machine.according to trial and error. The fusion power and fusion time have been changed.

After the fusion splicing, the air holes of PCF collapsed and an elliptical air cavity was created in the tapering region. The schematic diagram and image of the microscope of the MZI are shown in Fig. 1

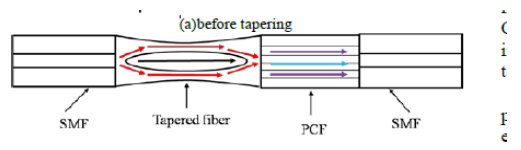

(a)

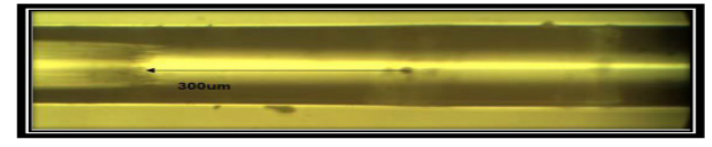

(b)

Fig. 1. schematic and microscope image of MZI.

As we known, the output intensity of this interferometer is given by [20]:

Where $I$ represent the intensity of the interference signal, $\lambda$ is the wavelength, $L$ denotes the interference length, $\Delta n$ eff denotes the effective refractive index difference between two interference arms. The shift in the output transmission spectrum is caused due to the optical path difference $\Delta n \operatorname{eff} L^{(14,16-19)}$. The MZI after tapering is based on the combination of two types of in line-fiber Mazh-Zehnder interferometer ${ }^{(14-16,18,19)}$.

The interference in the air cavity. At the collapsing region, the beam of the input light is split into two beams. One of these beams transmits through the silica wall, while the other travels along the internal air cavity, and the interference obtains when two output beams recombine at the collapsing region on the other side of the air cavity. In this case, the effective refractive index difference (marked as $\Delta$ ) can be given as: $\Delta=-$, where and Refer to the effective RI of the silica wall and the air cavity, respectively.

The interference in the photonic crystal fiber. The incident beam gradually expands in the collapsing region between the air cavity and the PCF, and lots of high order modes are excited, including the cladding modes. The high order modes are recombined by the collapsing section on the second side of photonic crystal fiber and interference with the fundamental core mode. Thus, the effective refractive index difference (marked as $\Delta$ ) can be expressed as: $\Delta=-$, where and Refer to the effective RI of the core mode and the cladding mode of the photonic crystal fiber. Finally, and, are sensitive to the external refractive index while and Are not. So $\Delta$ of both types of interference will change with external refractive index.

sample testing:

The Absorption Spectrum Measurement of the Blood Sample

A spectrophotometer (SP-8001) which runs in wavelength range from (190-1100) $\mathrm{nm}$ has been used to find the UV-Visible absorption spectrum of blood sample. Figure 6. shows the set-up of UV-Visible-IR Spectrophotometer. Sample of blood had been filled in the quartz cuvette and the absorption spectrum found .The selection of the light sources is done according to absorption spectrum have been obtained.

The Refractive Indices Measurement of the Blood Samples

The refractive indices of health and patient blood samples were measured by using Abbe refractometer.

The experimental measuring can be calculated by using the following equation:

Where is the experimental RI value measured at temperature, $\mathrm{T}$.

In this study, difference blood samples lead to change the modes effective refractive index of the clad of the fiber. This change of the cladding refractive index (ncladding) due to the change in refractive index value of blood samples changes transmission intensity.

\section{Results and Discussion}

The Absorption Spectra for the Blood Sample

The absorption spectra for blood sample and 
urine sample are measured by using (T60 UV-VIS) Spectrophotometer to select the suitable wavelength of laser source for the detection set-up. A figure 2.shows the absorption spectra for the blood sample.

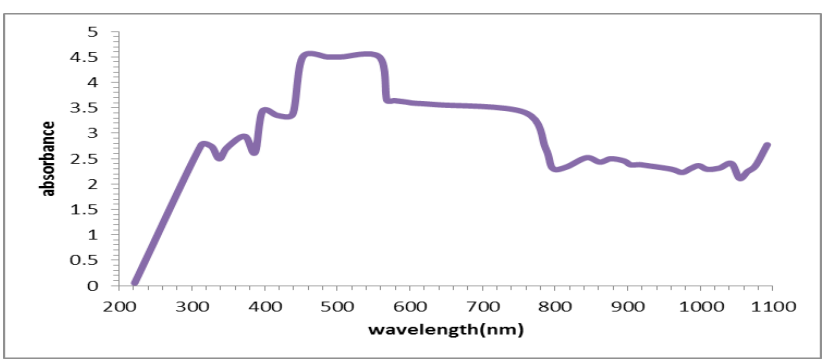

Figure 2.the maximum absorbance of the blood sample is for wavelengths in the range $\mathbf{( 4 7 0 - 5 9 0 )} \mathbf{n m}$. Thus, using green laser $532 \mathrm{~nm}$ as laser source for laser biosensor for blood test is quite convenient.

Detection of Beta Thalassemia Trait by Using Laser Biosensor

As mention previously laser source is used $(532 \mathrm{~nm}$ wavelength, power $12.2 \mathrm{nw}$ ) which chosen according to the blood absorption spectrum. PCF (LMA-10) with $1.5 \mathrm{~cm}$ length is used in this experiment. The transmitted intensity of this laser without any blood sample is shown in the figure below:

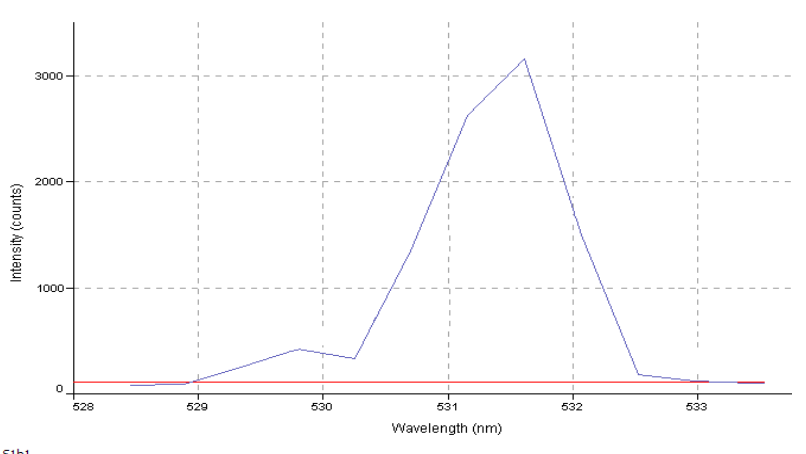

s1b1

Fig. 3.The output spectrum of the green laser $532 \mathrm{~nm}$.

The maximum output reference intensity before immersing sensor with any blood sample was 3159 . Blood samples were taken from healthy and patients with beta thalassemia trait. The patient samples were diagnostic from pathological analysis. According to the changes in the intensity modulation of the transmission laser, different blood tests are investigated. The sensor is embedded with these blood samples and the transmission spectra are shown in Fig. 4

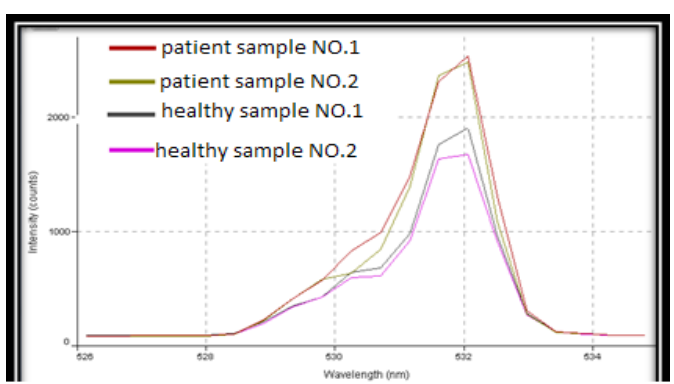

Figure (4): transmission spectra of laser biosensor for blood samples at $1.5 \mathrm{~cm}$ LMA-10 PCF length.

The refractive indices and absorbance of each blood samples which is measured according to the reference intensity and the maximum intensity are tabulated in Table 1.

Table 1. The values of maximum intensity and absorbance for the blood samples with different $\mathbf{H b}$ concentration at (1.5) cm PCF length.

\begin{tabular}{|l|l|l|l|}
\hline $\begin{array}{l}\text { Blood } \\
\text { sample }\end{array}$ & $\begin{array}{l}\text { Refractive } \\
\text { Index n }\end{array}$ & $\begin{array}{l}\text { Maximum } \\
\text { intensity } \mathbf{I}_{\mathbf{0}} \\
\text { (a.u) }\end{array}$ & $\begin{array}{l}\text { Absorbance } \\
\text { A=Log] } \\
\text { 【Iref/Io } \rrbracket\end{array}$ \\
\hline Patient No.1 & 1.34354 & 2488 & 0.1036 \\
\hline Patient No.2 & 1.34591 & 2470 & 0.1068 \\
\hline $\begin{array}{l}\text { Healthy } \\
\text { person No.1 }\end{array}$ & 1.34893 & 1886 & 0.224 \\
\hline $\begin{array}{l}\text { Healthy } \\
\text { person No.2 }\end{array}$ & 1.35192 & 1619 & 0.2903 \\
\hline
\end{tabular}

As a final result, the calibration curve of laser biosensor for detection beta thalassemia trait is shown in Figure below.

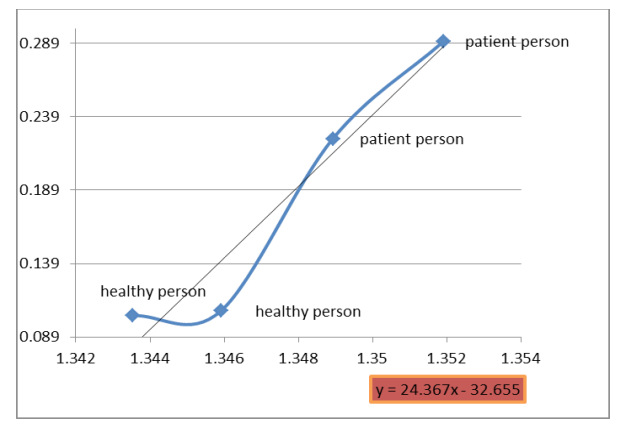

Fig. 5. The calibration curve of laser biosensor for detection beta thalassemia trait at $1.5 \mathrm{~cm}$ LMA-10 PCF length (sensitivity $=24.367$ ABS/RIU). 
It's shown from Figure 5 that the presence of beta thalassemia trait leads to decreasing the refractive index and then decreasing in the absorbencies of the blood samples to (0.1036 and 0.1068$)$ comparing with absorbencies of the blood samples of healthy people (0.224 and 0.2903).

\section{Conclusions}

According to the results found in this study, by using $1.5 \mathrm{~cm}$ PCF length, a fabricated laser biosensor based on MZI for detection beta thalassemia trait shows sensitivity of $24.367 \mathrm{ABS} / \mathrm{RIU}$.the maximum absorbance of the normal blood sample is at wavelengths from (470-590) $\mathrm{nm}$. The presence of beta thalassemia trait shows a decreasing in the refractive index of blood sample. Moreover, the absorbance of blood sample for healthy people showed higher values than those for the sample with anemia.

\section{References}

1. L. E. Savastano, Q. Zhou, A. Smith, K. Vega, C. Murga-Zamalloa, D. Gordon, J. McHugh, L. Zhao, M. M. Wang, A. Pandey, B. G. Thompson, J. Xu, J. Zhang, Y. E. Chen, E. J. Seibel, and T. D. Wang, "Multimodal laser-based angioscopy for structural, chemical and biological imaging of atherosclerosis," Nature Biomedical Engineering, vol. 1, no. 2, 2017.

2. J. Pollet, F. Delport, K. P. F. Janssen, D. T. Tran, J. Wouters, T. Verbiest, and J. Lammertyn, "Fast and accurate peanut allergen detection with nanobead enhanced optical fiber SPR biosensor," Talanta, 2011.vol. 83, no. 5, pp. 1436-1441,

3. A. Sadana and N. Sadana, Biomarkers and Biosensors: Detection and Binding to Biosensor Surfaces and Biomarkers Applications. 2014.

4. Y. Tan, A. Chu, and B. T. Cunningham, "Distributed feedback laser biosensor noise reduction," in 2012 IEEE Photonics Conference, IPC 2012, 2012.

5. X. J. Hao, X. H. Zhou, Y. Zhang, L. H. Liu, F. Long, L. Song, and H. C. Shi, "Melamine detection in dairy products by using a reusable evanescent wave fiber-optic biosensor," Sensors and Actuators, B: Chemical, , 2014 vol. 204, pp. 682-687.

6. Y. Tian, W. Wang, N. Wu, X. Zou, and X. Wang, "Tapered optical fiber sensor for label-free detection of biomolecules," Sensors, 2011. vol. 11, no. 4, pp. 3780-3790,
7. P. D’Orazio, "Biosensors in clinical chemistry 2011 update," Clinica Chimica Acta, 2011. vol. 412, no. 19-20. pp. 1749-1761,

8. S. Pevec and D. Donlagic, "Miniature all-fiber Fabry-Perot sensor for simultaneous measurement of pressure and temperature," Applied Optics, , 2012. vol. 51, no. 19 , p. 4536

9. H. S. Dhadwal, P. Kemp, J. Aller, and M. M. Dantzler, "Capillary waveguide nucleic acid based biosensor," Analytica Chimica Acta, vol. 501, no. 2, pp. 205-217, 2004.

10. M.Sura Huessein,Tahreer.Safa'a Mansour,H.Yousif Ibrahim,M. Laith Tariq," Pulse Compression by using 7 and 19 cell Photonic Crystal Fiber" Journal of Engineering and Applied Sciences, ,2018. vol 13, Issue 17,pp.7198-7204.

11. Y. Zhao, X. Li, X. Zhou, and Y. Zhang, "Review on the graphene based optical fiber chemical and biological sensors," Sensors and Actuators B: Chemical, 2016. vol. 231, pp. 324-340,

12. C. M. Li, C. W. Chan, J. S. Horng, J. M. Hsu, and C. L. Lee, "Fiber-optic twist sensor based on a tapered fiber Mach-Zehnder interferometer," in Pacific Rim Conference on Lasers and Electro-Optics, CLEO Technical Digest, 2013.

13. X. Dong, "Intensity-modulated optical fiber sensors based on chirped-fiber bragg gratings," Photonic Sensors, 2011. vol. 1, no. 3. pp. 251-259,

14. C. Ge, M. Lu, S. George, C. J. Wagner, J. Zheng, A. Pokhriyal, J. G. Eden, and B. T. Cunningham, "External cavity laser biosensor," in 2012 IEEE Photonics Conference, 2012, IPC 2012, pp. 151152.

15. Aia T.Yhia,Tahreer S.Mansour,Yousif I.Hammadi,"Tunable Notch Fiber Bragg Grating Optical Filter “,Journal of Engineering and Applied Sciences, 2018 vol 13,Issue 17,pp. 7450-7458.,

16. Faraqid Q. Mohammed and Tahreer S. Mansour," Design and Implementation Tunable Band Pass Filter based on PCF Air Micro-cavity FBG FabryPerot Resonator "2019 Vol.18,No.1, pp.13-23,

\section{Faraqid \\ Q. $\quad$ Mohammed}

Tahreer S. Mansoor,Ahmed W. Abdulwahhab," A tunable Mach-Zehnder interferometer based on dual micro-cavity photonic crystal fiber for load measurement", Photonic Network Communications, june 2019. 
18. Fareed F. Rashid, Sara J. Belal, Layla M. Alameri, Tahreer S. Mansour."Laser Biosensor For The Detection of The Human Blood Hemoglobin Concentration" Proc. of the Eighth Intl. Conf. on Advances in Bio-Informatics, Bio-Technology and Environmental Engineering - ABBE 2019.

19. N. Ali, B. Moiz, W. Bin Azhar, N. Zaidi, and R. Memon, "Carrier detection for beta-thalassemia trait in general Pakistani population: a way forward," Hematology, 2012. vol. 17, no. 4, pp. 237-240,

20. W. Yu, T. Lang, J. Bian, and W. Kong, "Labelfree fiber optic biosensor based on thin-core modal interferometer," Sensors and Actuators, B: Chemical, 2016. vol. 228, pp. 322-329, 